\title{
Modeling particle acceleration and non-thermal emission in supernova remnants
}

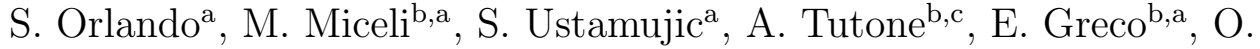 \\ Petruk $^{\mathrm{d}}$, F. Bocchino ${ }^{\mathrm{a}}$, G. Peres ${ }^{\mathrm{b}, \mathrm{a}}$ \\ ${ }^{a}$ INAF - Oss. Astronomico di Palermo, Piazza del Parlamento 1, 90134 Palermo, Italy \\ ${ }^{b}$ Dip. di Fis. e Chim., Università di Palermo, Via Archirafi 36, 90123 Palermo, Italy \\ ${ }^{c} I N A F$ - IASF, Via Ugo La Malfa 153, 90146 Palermo, Italy \\ ${ }^{d}$ Inst. for Appl. Probl. in Mech. and Math., Naukova Street, 3-b Lviv 79060, Ukraine
}

\begin{abstract}
According to the most popular model for the origin of cosmic rays (CRs), supernova remnants (SNRs) are the site where CRs are accelerated. Observations across the electromagnetic spectrum support this picture through the detection of non-thermal emission that is compatible with being synchrotron or inverse Compton radiation from high energy electrons, or pion decay due to proton-proton interactions. These observations of growing quantity and quality promise to unveil many aspects of CRs acceleration and require more and more accurate tools for their interpretation. Here, we show how multidimensional MHD models of SNRs, including the effects on shock dynamics due to back-reaction of accelerated CRs and the synthesis of non-thermal emission, turned out to be very useful to investigate the signatures of CRs acceleration and to put constraints on the acceleration mechanism of high energy particles. These models have been used to interpret accurately observations of SNRs in various bands (radio, X-ray and $\gamma$-ray) and to extract from them key information about CRs acceleration.
\end{abstract}

Keywords: cosmic rays, magnetohydrodynamics (MHD), radiation mechanisms: non-thermal, shock waves, ISM: supernova remnants

Email address: salvatore.orlando@inaf.it (S. Orlando) 


\section{Introduction}

Many lines of evidence suggest that supernova remnants (SNRs) are the site where CRs diffusive shock acceleration (DSA) occurs. Roughly $10 \%$ of the supernova (SN) energy would be required to be transmitted to CRs to satisfy observations. In fact, observations in various bands support this idea through the detection of non-thermal emission that is compatible with being synchrotron radiation from CR electrons. Conversely, the direct evidence of CR ions is difficult to find because they do not radiate efficiently; some hints of their existence come from the analysis of $\gamma$-rays.

On the other side, the effects of CR ions on the dynamics of the shock waves (and, therefore, on the thermal emission from the shell of SNRs in different wavelength bands) are often invoked as indirect evidence for their existence. A possible signature of $\mathrm{CR}$ ions reported in the literature is the separation between the forward shock (FS) and the contact discontinuity (CD) in young SNRs; this separation should be smaller than expected, if CRs acceleration is efficient (e.g. Ferrand et al. 2010). Furthermore CR ions affect the evolution of the magnetic field and the signature of their presence can be found in the non-thermal emission from the electrons in different bands (radio, X-rays, $\gamma$-rays; e.g. Ferrand et al. 2014).

The numerical simulations involving CRs acceleration at shock fronts of SNRs is studied by following two complementary approaches. The first is based on highly detailed models implemented with particle-in-cell (PIC) and hybrid (kinetic ions-fluid electrons) simulations (e.g. Spitkovsky 2008; Caprioli and Spitkovsky 2014a,b; Pohl et al. 2020). These models are based on first principles and consider few or no parameters/approximations. However, they require high computational resources and they have limited dynamical ranges. For instance, these models cannot simulate the whole remnant interacting with the inhomogeneous interstellar medium (ISM) or describe the complete evolution from the SN event to the full-fledged SNR.

The second approach is based on three-dimensional (3D) (magneto)hydrodynamic (HD/MHD) simulations coupled with semi-analytic models which provide a general description of the CRs acceleration (e.g. Ferrand et al. 2010; Orlando et al. 2012). This approach is less accurate in the prescription of CRs than the previous one but allows to simulate the evolution of the whole remnant from the SN explosion to its interaction with the inhomogeneous and magnetized ISM. These models include the microphysics relevant for the evolution of a SNR (e.g. magnetic-field-oriented thermal con- 
duction 1 , deviations from equilibrium of ionization and from electron-proton temperature equilibration, radiative cooling, etc.) and they are constrained by multi- $\lambda$ observations.

While the first approach (based on PIC, hybrid simulations) is mainly oriented toward an accurate description and a deep physical insight of the microscopic mechanisms for particle acceleration, the second one (based on HD/MHD simulations coupled with semi-analytic models of particle acceleration) is mostly oriented toward a description of the macroscopic effects of particle acceleration on the dynamics and evolution of SNRs, especially during the interaction of the remnants with the inhomogeneous ISM. This latter approach can be very powerful for the interpretation of observations, and for obtaining information and constraints on the mechanisms of particle acceleration (e.g. Bocchino et al. 2011; Miceli et al. 2016).

Here, we discuss some of the recent achievements obtained using the second approach, the capabilities and limitations of the HD/MHD models describing the macroscopic effects of particle acceleration, and the methodologies generally adopted to study and use these models for the interpretation of observations in different bands. We limit the discussion to models mostly developed by our group; so we warn the reader that the review is highly biased on results we obtained in the last years.

\section{Modeling the macroscopic effects of particle acceleration on the dynamics of SNRs}

Models of SNRs, which include the effects of particle acceleration, have to describe all the features characterizing a remnant (multiple shocks, HD instabilities developing during the evolution, interaction with inhomogeneities of the ISM, etc.) and to synthesize the thermal and non-thermal emission in various bands. Examples of these models are those produced with the CRhydro-NEI (ChN) code (Lee et al. 2008, 2012), a one-dimensional (1D) code appropriate to describe young to middle-aged SNRs. It includes nonlinear DSA physics, and describes the CR back-pressure, the particle escape, and the magnetic turbulence generation. The output of the code (multi- $\lambda$ spec-

\footnotetext{
${ }^{1}$ In the presence of an organized magnetic field, the thermal conduction is highly anisotropic and it can be extraordinarily reduced in the direction transverse to the field. In this case, the models describe a magnetic-field-oriented thermal conduction (e.g. Orlando et al. 2008).
} 
tra, spatial variation of emission, thermal and non-thermal emission, etc.) can be compared directly with observations, thus providing a key for the interpretation of the data (and also to get a feedback on the models).

Nevertheless, although very sophisticated and rich in physics, the ChN code is $1 \mathrm{D}$ and, therefore, it is not adapt to describe the asymmetries which develop during the remnant evolution and the complex interaction of the SNR with the inhomogeneous ISM. In these latter cases a full 3D description is required. Ferrand et al. (2010) developed a 3D model of SNR coupled with a semi-analytical kinetic model of shock acceleration (Blasi 2002, 2004) by means of an effective adiabatic index which depends on time. The nonlinear model of DSA includes the escape of particles with the highest energy upstream of the shock and the effect of Alfvén wave heating in the precursor. With their model, the authors have studied the time-dependent compression of the region between the FS and the reverse shock (RS) due to the backreaction of accelerated particles, for different values of the injection efficiency. They found that the density profiles and the thickness of the mixing region depend critically on the injection level of particles.

Moreover, this model provides a theoretical support to the idea that a greater shock compression ratio and a thinner shell of shocked ISM are direct consequences of the energy losses to CRs at the FS. Evidence of thinner shell of shocked ISM have been found in some SNRs (e.g. Tycho and SN 1006), suggesting the presence of efficient CRs acceleration (e.g. Blondin and Ellison 2001; Miceli et al. 2009; Ferrand et al. 2010). Some authors, however, have raised doubts if the CRs energy losses at shock fronts alone can naturally explain observations as those of Tycho and SN 1006 (e.g. Rakowski et al. 2011; Orlando et al. 2012).

In fact, the observations show that, in general, remnant outlines are characterized by several protrusions by ejecta fragments. These features may be explained as due to a thinner shell of shocked ISM caused by energy losses to CRs (e.g. Cassam-Chenaï et al. 2008; Rakowski et al. 2011). However, the number of protrusions in regions dominated by thermal emission is more or less the same as in regions dominated by non-thermal emission and this evidence suggests that the occurrence of protrusions cannot be explained in terms of energy losses to CRs alone. In addition, extreme energy losses are needed to allow a significant fraction of the ejecta to approach or even overtake the FS to form protrusions. For instance, Wang and Chevalier (2001) have shown that an extreme effective adiabatic index of the order of 1.1 would be necessary to produce evident protrusions in a remnant outline. Further- 
more, in the case of $\mathrm{SN} 1006$, the ratio of $\mathrm{FS}$ to $\mathrm{CD}$ radii is almost the same at all azimuthal angles (Miceli et al. 2009), in regions dominated by non-thermal emission (where CRs acceleration is efficient) and in those dominated by thermal emission (where the CRs acceleration efficiency should be negligible). This is in stark contrast with the predictions that the ratio of FS to $\mathrm{CD}$ radii should be the minimum in regions of efficient CRs acceleration.

All these lines of evidence suggest that FS-CD separation may be misleading as indicator for the efficiency of CRs acceleration. Rakowski et al. (2011) suggested that the ejecta structure of the explosion itself can play a role in determining the frequent occurrence of protrusions (even in regions with no evident CRs acceleration). In other words, the observed structure of the mixing region between the FS and the RS may originate in the intrinsic structure of the ejecta.

\section{Modeling the evolution from the SN to the SNR including the CRs acceleration}

The difficulties of models accounting only for the back-reaction of accelerated CRs in the interpretation of observations have stimulated the development of 3D HD/MHD models which describe accurately the structure of the ejecta originating from the SN explosion. These models describe the evolution from the SN to the development of its full-fledged remnant (e.g. Orlando et al. 2015, 2016; Wongwathanarat et al. 2017; Ferrand et al. 2019; Ono et al. 2020; Orlando et al. 2020a; Tutone et al. 2020; Orlando et al. 2020b). The strategy is based on the coupling between SN models and SNR models. The SN models provide the initial conditions for the SNR simulations few hours after the SN event and, among other things, include: the explosive nucleosynthesis through a nuclear reaction network, the energy deposition due to radioactive decays of isotopes synthesized in the $\mathrm{SN}$, the gravitational effects of the central compact object, and the fallback of material on the compact object (e.g. Wongwathanarat et al. 2017; Ono et al. 2020). The pre-SN structure of the stellar progenitor is derived by available stellar evolution codes. The SNR models include: the effects of an ambient magnetic field, the radiative cooling, the deviations from equilibrium of ionization and from electron-proton temperature equilibration, the heating due to radioactive decay of unstable elements, and the back-reaction of accelerated CRs (e.g. Orlando et al. 2016, 2020b). The SNR models also describe the geome- 
try and density structure of the ambient medium through which the remnant expands on the base of constraints from multi- $\lambda$ observations.

This approach also includes the synthesis of both thermal and non-thermal emission from the models for a qualitative and quantitative comparison of the model results with specific observations (e.g. Orlando et al. 2007, 2011; Miceli et al. 2019). This is an essential step to disentangle the effects of CRs acceleration from other effects as, for instance, the intrinsic ejecta structure reflecting the asymmetries in the SN explosion and the interaction of the remnant with the inhomogeneous ISM.

In the following, we discuss a few examples to show how the above approach can be used to extract various pieces of information on the acceleration of paticles in SNRs from the comparison of model results with observations. More specifically, in Sect. 3.1, we discuss the need to identify the effects of $\mathrm{CR}$ acceleration on the structure of the mixing region in SNRs and to disentangle these effects from those of the intrinsic structure of the ejecta. In Sect. 3.2, we highlight the importance of modeling the interaction of SNRs with the inhomogeneous ISM (in particular with atomic/molecular clouds), since the interaction sites provide excellent opportunities to prove unambiguously CRs acceleration (being the densities in clouds high enough to allow the $\gamma$-ray emission to be dominated by protons) and to constrain the energetics of particle accelerators. Finally, in Sect. 3.3, we consider the process of particle acceleration during the transition from the phase of SN to that of SNR, a time in which the particles start to be accelerated and any variations of the acceleration process in these early times after the SN may be reflected in the shape of $\gamma$-ray spectra in young and middle-age SNRs (e.g. Petruk et al. 2017).

\subsection{Back-reaction of accelerated CRs and clumping of ejecta}

The numerical approach described above was used to investigate the role played by back-reaction of accelerated CRs and by the intrinsic structure of the ejecta in determining the ratio of FS to CD radii (Orlando et al. 2012). Figures 1 and 2 show the results of models either with or without an initial small-scale structure of ejecta, respectively. The models assume that the effects of the back-reaction of accelerated CRs is modulated by the obliquity angle and that they are more effective at parallel shocks where the adiabatic index reaches values as low as 1.1 (in models without ejecta clumping, thus indicating extreme energy losses to accelerate the CRs) or 4/3 (in models including the ejecta clumping). 

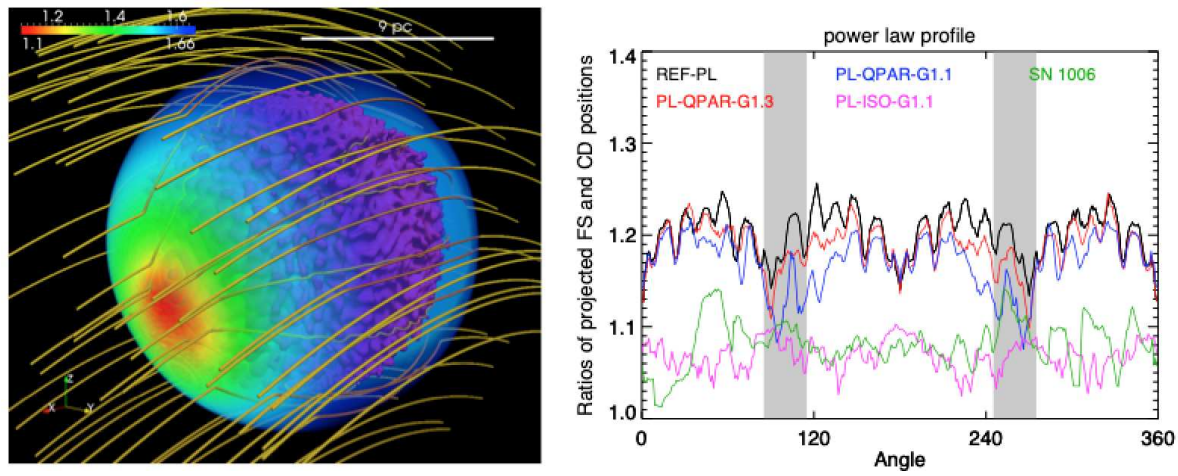

Figure 1: 3D rendering of the spatial distribution of the effective adiabatic index (left panel) and azimuthal profiles of the ratio between the FS and CD radii when the aspect angle is 90 degrees (right panel), for models including the back-reaction of accelerated CRs without the clumping of ejecta. The violet surface tracks the ejecta material, the yellow lines are sampled magnetic field lines. Black, red and blue profiles mark the results from the models; the green line marks the profile derived from observations of SN 1006 (Miceli et al. 2009). The gray areas in the right panel mark the regions where the acceleration of CRs is the largest. Figure adapted from Orlando et al. (2012).

Figure 1 shows that none of the models without ejecta clumping and with a dependence on the obliquity angle is able to reproduce the profile inferred from the observations of SN 1006 (green line). A model without ejecta clumping can reproduce the observations only if the effects of back-reaction of accelerated CRs are extreme (with adiabatic index 1.1) at all obliquity angles (see the purple profile in the upper right panel), a condition which is not realistic. In models including the ejecta clumping (Fig. 21), clumps and filamentary structures are evident within the remnant, and fingers of ejecta are close to or protrude beyond the main blast wave as observed by Rakowski et al. (2011) in SN 1006. Indeed, these models can naturally reproduce the observed azimuthal profile of the ratio between the FS and CD radii in SN 1006 (see right panel in Fig. (2). This study unambiguously shows that the FS-CD separation is a probe of the ejecta structure rather than a probe of the efficiency of CRs acceleration in young SNRs.

\subsection{Interaction of supernova remnant with interstellar clouds}

The interaction of SNRs with atomic or molecular clouds is quite common (a few examples are IC 443 and W48b) and it is important for the acceleration of CRs. For instance, MHD simulations of the shock-cloud interaction revealed a crucial role played by the magnetic field (Petruk et al. 2016, 2018): 

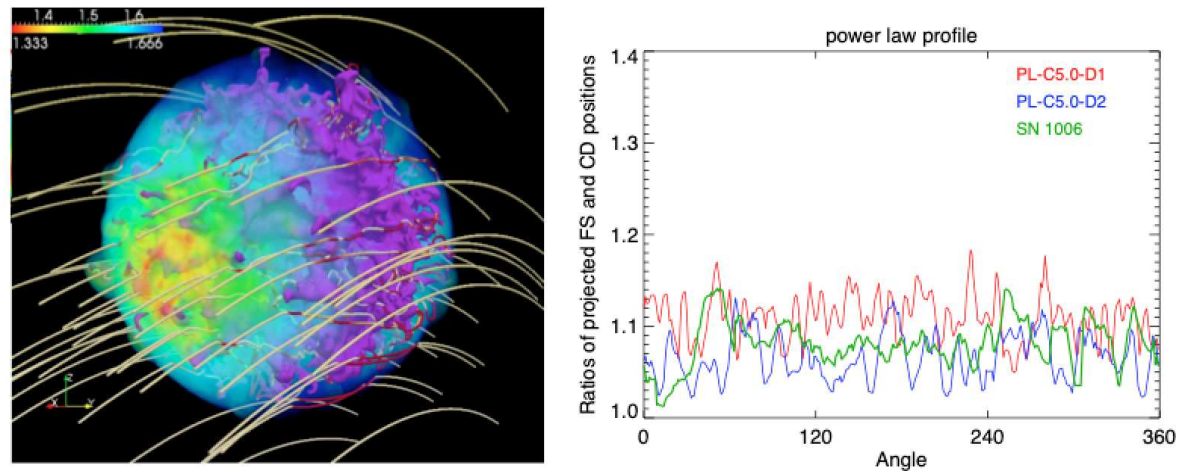

Figure 2: As in Fig. 1 but for models including the clumping of ejecta in addition to the back-reaction of accelerated CRs. Figure adapted from Orlando et al. (2012).

the tangential magnetic field component prevents a large compression of postadiabatic shocks visible in the pure HD simulations. This effect may lower the $\gamma$-ray flux arising from the shock-cloud interaction region. IC 443 is an example of remnant interacting with clouds; it is a strong $\gamma$-ray source and one of the few remnants providing evidence for hadronic CRs acceleration. Observations show a diffuse $\gamma$-ray emission coincident with the FS of IC 443; a clear enhancement of $\gamma$-ray emission is evident in the northeast (NE) rim where the remnant is interacting with a molecular cloud.

SN 1006 is another remnant showing evidence of interaction with an atomic cloud. This SNR is an interesting target to study particle acceleration because of the bilateral morphology of its non-thermal emission which reflects efficient particle acceleration in the NE and southwest (SW) limbs bright in the radio, X-ray and $\gamma$-ray bands. XMM observations of this remnant revealed a sharp indentation in the SW limb and several signatures for a shock-cloud interaction (Miceli et al. 2014) in a region corresponding to the position of an H I cloud. A clear proof of the shock-cloud interaction is provided by the azimuthal profile of the cutoff energy which has a minimum in coincidence with the indentation.

The shock-cloud interaction was investigated through 3D MHD simulations, including the back-reaction of particle acceleration, and describing the interaction of the remnant with the H I cloud (Miceli et al. 2016). Fig. 3 shows the XMM observation of the SW limb and the synthetic non-thermal $\mathrm{X}$-ray image derived from the best-fit model. The model roughly describe the distribution of surface brightness and the indentation observed in XMM 

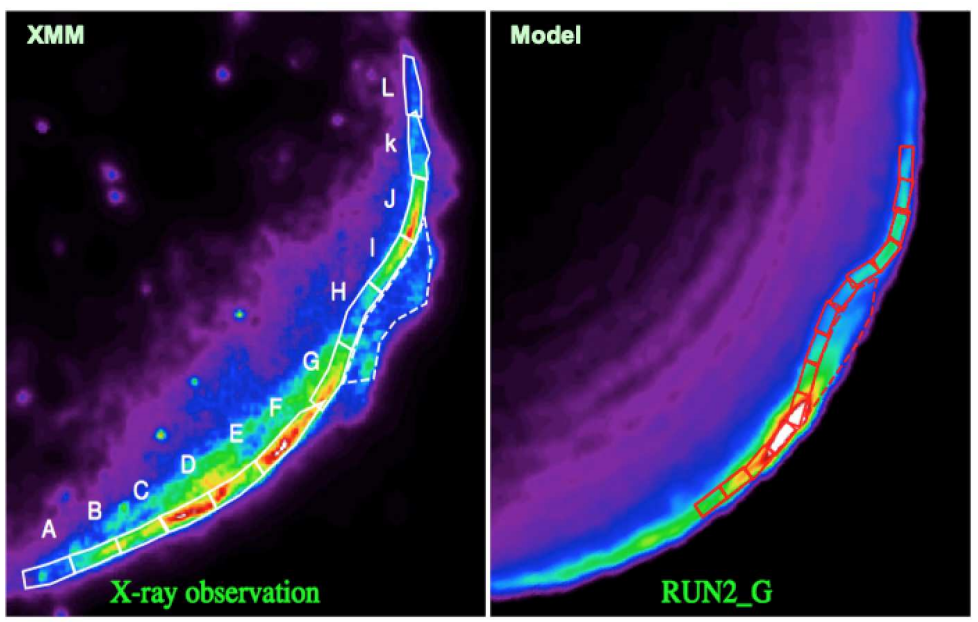

Figure 3: XMM-Newton (on the left) and modeled (on the right) images of the SW limb of SN 1006 in the $[2,4.5] \mathrm{keV}$ band. The images show the regions selected for the spectral analysis of the rim. Figure adapted from Miceli et al. (2016).

observations. Also, the model is able to reproduce the azimuthal profile of cutoff energy inferred from observations, and in particular the drop in energy in coincidence with the indentation (see Fig. 4). The synthesis of the hadronic and leptonic emission in the $\gamma$-ray band from the models and the comparison of the model results with observations collected by HESS and FERMI allowed to constrain the total hadronic energy to $\approx 5 \times 10^{49} \mathrm{erg}$, a value confirmed later by Condon et al. (2017) who found that this hadronic energy is conceivable to explain the $\gamma$-ray emission in the SW region of SN 1006.

\subsection{Particle acceleration in very young SNRs: the case of $S N 1987$ A}

Among other things, very young SNRs offer the opportunity to study the process of particle acceleration during the transition from the phase of SN to that of SNR. One of the best case to study is certainly SN 1987A. The SN was observed in February 1987 and, since then, was continuously monitored at all wavelength bands. About three years later the blast wave started to interact with a dense circumstellar nebula consisting of a dense equatorial ring and an extended H II region.

The model approach described above was adopted to investigate the evolution of SN 1987A from the immediate aftermath of the core-collapse of the progenitor star to the subsequent interaction of its remnant with the nebula. The model includes the synthesis of thermal X-ray emission and non-thermal 


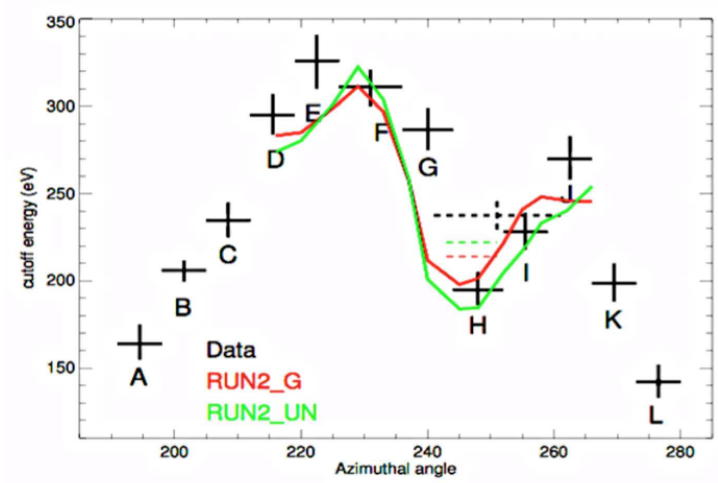

Figure 4: Azimuthal variations of the synchrotron cutoff energy. The black crosses show the best fit values obtained from the analysis of the X-ray spectra extracted from the regions marked in Fig. 3. The green/red curves show the values derived from two MHD models. Figure adapted from Miceli et al. (2016).

radio emission to compare the model results with observations of SN 1987A. The observed (and modeled) X-ray (in both the soft and hard bands) and radio lightcurves show that, few years after the $\mathrm{SN}$, both X-ray and radio fluxes suddenly increased after the impact of the blast wave with the H II region (see Fig. [5). In the subsequent years the X-ray and radio fluxes have continued to rise together with almost constant slope. About 14 years after the SN the soft X-ray flux has steepened still further due to the interaction with the dense equatorial ring, contrary to the hard X-ray and radio lightcurves which continued to rise with almost constant slope.

Thanks to the model it was possible to explain the origin of the X-ray and radio emission. In fact, the hard X-ray emission is almost insensitive to the interaction of the blast wave with the ring because the latter is very dense and the shocked ring material emits mainly in the soft X-ray band. Conversely, most of the contribution to the hard X-ray emission originates from shocked material from the HII region. This determines the sudden rise of the soft X-ray emission during the interaction with the ring and an almost constant slope of the hard X-ray emission (Orlando et al. 2015). The 3D MHD model of SN 1987A also provided a natural explanation for the origin of the observed trend of the radio emission (similar to that of the hard $\mathrm{X}$-ray emission). In particular, the model can reproduce the observed radio lightcurves if the radio flux originating from the RS is partially suppressed (see Fig. 5, Orlando et al. 2019). As a result, the radio emission originates 

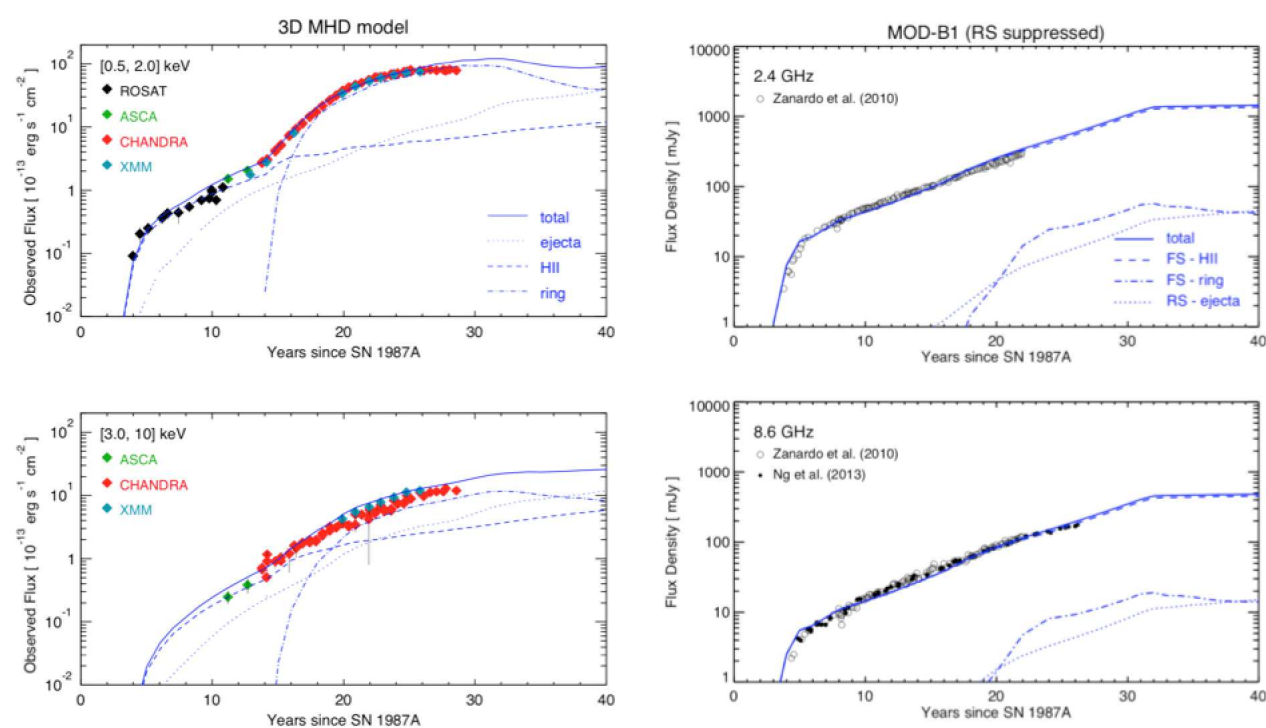

Figure 5: Observed (symbols) and synthetic (lines) X-ray lightcurves in the $[0.5,2] \mathrm{keV}$ (upper left panel) and $[3,10] \mathrm{keV}$ (lower left panel) bands and radio flux densities at $2.4 \mathrm{GHz}$ (upper right panel; Zanardo et al. 2010) and $8.6 \mathrm{GHz}$ (lower right panel; Zanardo et al. 2010; Ng et al. 2013). Solid lines show the synthetic lightcurves; dotted, dashed, and dotdashed lines indicate the contribution to emission from the shocked ejecta, the shocked plasma from the H II region, and the shocked plasma from the ring, respectively. The model assumes that the radio emission from the RS is reduced by two orders of magnitude. Figure adapted from Orlando et al. (2019).

mostly from the FS traveling through the H II region (the contribution from the FS traveling through the ring is at least an order of magnitude lower) and this explains why the radio emission is insensitive to the interaction of the blast with the ring. The analysis of possible mechanisms for the suppression of emission from the RS has shown that synchrotron self-absorption and free-free absorption have negligible effects on the emission. Thus the model suggests that the emission from the RS at radio frequencies might be limited by highly magnetized ejecta (Orlando et al. 2019).

\section{Summary and conclusions}

In this contribution, we discussed how models describing the evolution of SNRs can include the macroscopic effects of particle acceleration at shock fronts, discussing possible approaches and methodologies. These models do 
not describe in detail the physics of particle acceleration as PIC/hybrid simulations based on first principles do (e.g. Caprioli and Spitkovsky 2014a, b) . On the other side, they allow to explore large dynamical ranges, describing the evolution of SNRs (since the SN explosion) and their interaction with the inhomogeneous ISM (e.g. Orlando et al. 2015, 2016).

Current limitations of these models include an accurate description of the back-reaction of accelerated particles which requires input from first principle simulations. In recent years, many authors have stressed that identifying the signature of CRs acceleration requires to link SNRs to their parent SN explosions and progenitor stars. In fact, establishing this connection is important to describe accurately the structure of ejecta which may be characterized by anisotropies developed soon after the core-collapse and the structure of the circumstellar medium sculpted by the winds of the progenitor star. This is an essential step to disentangle the effects of CRs acceleration from other effects determining the structure and morphology of SNRs in view of an accurate comparison of model results with multi- $\lambda$ observations of SNRs.

Acknowledgements. We thank the anonymous referee for useful suggestions that have allowed us to improve the paper. We acknowledge financial contribution from the INAF mainstream program and from the agreement ASI-INAF n.2017-14-H.O.

\section{References}

Blasi, P., 2002. A semi-analytical approach to non-linear shock acceleration. Astroparticle Physics 16, 429-439. doi 10.1016/S0927-6505(01)00127-X.

Blasi, P., 2004. Nonlinear shock acceleration in the presence of seed particles. Astroparticle Physics 21, 45-57. doi:10.1016/j . astropartphys .2003.10.008.

Blondin, J.M., Ellison, D.C., 2001. Rayleigh-Taylor Instabilities in Young Supernova Remnants Undergoing Efficient Particle Acceleration. Astrophys. J. 560, 244-253. doi:10.1086/322499.

Bocchino, F., Orlando, S., Miceli, M., Petruk, O., 2011. Constraints on the local interstellar magnetic field from non-thermal emission of SN1006. Astron. \& Astrophys. 531, A129. doi:10.1051/0004-6361/201016341. 
Caprioli, D., Spitkovsky, A., 2014a. Simulations of Ion Acceleration at Non-relativistic Shocks. I. Acceleration Efficiency. Astrophys. J. 783, 91. doi:10.1088/0004-637X/783/2/91.

Caprioli, D., Spitkovsky, A., 2014b. Simulations of Ion Acceleration at Nonrelativistic Shocks. II. Magnetic Field Amplification. Astrophys. J. 794, 46. doi:10.1088/0004-637X/794/1/46.

Cassam-Chenaï, G., Hughes, J.P., Reynoso, E.M., Badenes, C., Moffett, D., 2008. Morphological Evidence for Azimuthal Variations of the CosmicRay Ion Acceleration at the Blast Wave of SN 1006. Astrophys. J. 680, 1180-1197. doi:10.1086/588015.

Condon, B., Lemoine-Goumard, M., Acero, F., Katagiri, H., 2017. Detection of Two TeV Shell-type Remnants at GeV Energies with FERMI LAT: HESS J1731-347 and SN 1006. Astrophys. J. 851, 100. doi:10.3847/1538-4357/aa9be8.

Ferrand, G., Decourchelle, A., Ballet, J., Teyssier, R., Fraschetti, F., 2010. 3D simulations of supernova remnants evolution including non-linear particle acceleration. Astron. \& Astrophys. 509, L10. doi:10.1051/0004-6361/200913666.

Ferrand, G., Decourchelle, A., Safi-Harb, S., 2014. Three-dimensional Simulations of the Non-thermal Broadband Emission from Young Supernova Remnants Including Efficient Particle Acceleration. Astrophys. J. 789, 49. doi:10.1088/0004-637X/789/1/49.

Ferrand, G., Warren, D.C., Ono, M., Nagataki, S., Röpke, F.K., Seitenzahl, I.R., 2019. From Supernova to Supernova Remnant: The Threedimensional Imprint of a Thermonuclear Explosion. Astrophys. J. 877, 136. doi:10.3847/1538-4357/ab1a3d.

Lee, S.H., Ellison, D.C., Nagataki, S., 2012. A Generalized Model of Nonlinear Diffusive Shock Acceleration Coupled to an Evolving Supernova Remnant. Astrophys. J. 750, 156. doi:10.1088/0004-637X/750/2/156.

Lee, S.H., Kamae, T., Ellison, D.C., 2008. Three-dimensional Model of Broadband Emission from Supernova Remnants Undergoing Nonlinear Diffusive Shock Acceleration. Astrophys. J. 686, 325-336. doi:10.1086/591308. 
Miceli, M., Acero, F., Dubner, G., Decourchelle, A., Orlando, S., Bocchino, F., 2014. Shock-Cloud Interaction and Particle Acceleration in the Southwestern Limb of SN 1006. Astrophys. J. 782, L33. doi:10.1088/2041-8205/782/2/L33.

Miceli, M., Bocchino, F., Iakubovskyi, D., Orlando, S., Telezhinsky, I., Kirsch, M.G.F., Petruk, O., Dubner, G., Castelletti, G., 2009. Thermal emission, shock modification, and X-ray emitting ejecta in SN 1006. Astron. \& Astrophys. 501, 239-249. doi:10.1051/0004-6361/200811505.

Miceli, M., Orlando, S., Burrows, D.N., Frank, K.A., Argiroffi, C., Reale, F., Peres, G., Petruk, O., Bocchino, F., 2019. Collisionless shock heating of heavy ions in SN 1987A. Nature Astronomy 3, 236-241. doi:10.1038/s41550-018-0677-8.

Miceli, M., Orlando, S., Pereira, V., Acero, F., Katsuda, S., Decourchelle, A., Winkler, F.P., Bonito, R., Reale, F., Peres, G., Li, J., Dubner, G., 2016. Modeling the shock-cloud interaction in SN 1006: Unveiling the origin of nonthermal X-ray and $\gamma$-ray emission. Astron. \& Astrophys. 593, A26. doi:10.1051/0004-6361/201628725.

Ng, C.Y., Zanardo, G., Potter, T.M., Staveley-Smith, L., Gaensler, B.M., Manchester, R.N., Tzioumis, A.K., 2013. Evolution of the Radio Remnant of Supernova 1987A: Morphological Changes from Day 7000. Astrophys. J. 777, 131. doi:10.1088/0004-637X/777/2/131.

Ono, M., Nagataki, S., Ferrand, G., Takahashi, K., Umeda, H., Yoshida, T., Orland o, S., Miceli, M., 2020. Matter Mixing in Aspherical Corecollapse Supernovae: Three-dimensional Simulations with Single-star and Binary Merger Progenitor Models for SN 1987A. Astrophys. J. 888, 111. doi:10.3847/1538-4357/ab5dba.

Orlando, S., Bocchino, F., Miceli, M., Petruk, O., Pumo, M.L., 2012. Role of Ejecta Clumping and Back-reaction of Accelerated Cosmic Rays in the Evolution of Type Ia Supernova Remnants. Astrophys. J. 749, 156. doi:10.1088/0004-637X/749/2/156.

Orlando, S., Bocchino, F., Reale, F., Peres, G., Pagano, P., 2008. The Importance of Magnetic-Field-Oriented Thermal Conduction in the Interaction of SNR Shocks with Interstellar Clouds. Astrophys. J. 678, 274-286. doi:10.1086/529420, arXiv:arXiv:0801.1403. 
Orlando, S., Bocchino, F., Reale, F., Peres, G., Petruk, O., 2007. On the origin of asymmetries in bilateral supernova remnants. Astron. \& Astrophys. 470, 927-939. doi:10.1051/0004-6361:20066045.

Orlando, S., Miceli, M., Petruk, O., Ono, M., Nagataki, S., Aloy, M.A., Mimica, P., Lee, S.H., Bocchino, F., Peres, G., Guarrasi, M., 2019. 3D MHD modeling of the expanding remnant of SN 1987A. Role of magnetic field and non-thermal radio emission. Astron. \& Astrophys. 622, A73. doi:10.1051/0004-6361/201834487.

Orlando, S., Miceli, M., Pumo, M.L., Bocchino, F., 2015. Supernova 1987A: a Template to Link Supernovae to Their Remnants. Astrophys. J. 810, 168. doi:10.1088/0004-637X/810/2/168.

Orlando, S., Miceli, M., Pumo, M.L., Bocchino, F., 2016. Modeling SNR Cassiopeia A from the Supernova Explosion to its Current Age: The Role of Post-explosion Anisotropies of Ejecta. Astrophys. J. 822, 22. doi:10.3847/0004-637X/822/1/22.

Orlando, S., Ono, M., Nagataki, S., Miceli, M., Umeda, H., Ferrand, G., Bocchino, F., Petruk, O., Peres, G., Takahashi, K., Yoshida, T., 2020a. Hydrodynamic simulations unravel the progenitor-supernovaremnant connection in SN 1987A. Astron. \& Astrophys. 636, A22. doi:10.1051/0004-6361/201936718.

Orlando, S., Petruk, O., Bocchino, F., Miceli, M., 2011. Effects of nonuniform interstellar magnetic field on synchrotron X-ray and inverseCompton $\gamma$-ray morphology of supernova remnants. Astron. \& Astrophys. 526, A129. doi:10.1051/0004-6361/201015505.

Orlando, S., Wongwathanarat, A., Janka, H.T., Miceli, M., Ono, M., Nagataki, S., Bocchino, F., Peres, G., 2020b. The fully developed remnant of a neutrino-driven supernova: Evolution of ejecta structure and asymmetries in SNR Cassiopeia A. arXiv e-prints , arXiv:2009.01789.

Petruk, O., Kuzyo, T., Beshley, V., 2016. Post-adiabatic supernova remnants in an interstellar magnetic field: parallel and perpendicular shocks. Mon. Not. R. Astron. Soc. 456, 2343-2353. doi:10.1093/mnras/stv2746.

Petruk, O., Kuzyo, T., Orlando, S., Pohl, M., Miceli, M., Bocchino, F., Beshley, V., Brose, R., 2018. Post-adiabatic supernova remnants in an 
interstellar magnetic field: oblique shocks and non-uniform environment. Mon. Not. R. Astron. Soc. 479, 4253-4270. doi:10.1093/mnras/sty1750.

Petruk, O., Orlando, S., Miceli, M., Bocchino, F., 2017. Linking gammaray spectra of supernova remnants to the cosmic ray injection properties in the aftermath of supernovae. Astron. \& Astrophys. 605, A110. doi:10.1051/0004-6361/201730956, arXiv:1707.00136.

Pohl, M., Hoshino, M., Niemiec, J., 2020. PIC simulation methods for cosmic radiation and plasma instabilities. Progress in Particle and Nuclear Physics 111, 103751. doi:10.1016/j.ppnp.2019.103751.

Rakowski, C.E., Laming, J.M., Hwang, U., Eriksen, K.A., Ghavamian, P., Hughes, J.P., 2011. The Effect of a Cosmic Ray Precursor in SN 1006? Astrophys. J. 735, L21. doi:10.1088/2041-8205/735/1/L21.

Spitkovsky, A., 2008. Particle Acceleration in Relativistic Collisionless Shocks: Fermi Process at Last? Astrophys. J. 682, L5. doi:10.1086/590248.

Tutone, A., Orlando, S., Miceli, M., Ustamujic, S., Ono, M., Nagataki, S., Ferrand, G., Greco, E., Peres, G., Warren, D.C., Bocchino, F., 2020. Three-dimensional modeling from the onset of the SN to the full-fledged SNR. Role of an initial ejecta anisotropy on matter mixing. Astron. \& Astrophys. 642, A67. doi:10.1051/0004-6361/202038336.

Wang, C.Y., Chevalier, R.A., 2001. Instabilities and Clumping in Type IA Supernova Remnants. Astrophys. J. 549, 1119-1134. doi:10.1086/319439.

Wongwathanarat, A., Janka, H.T., Müller, E., Pllumbi, E., Wanajo, S., 2017. Production and Distribution of ${ }^{44} \mathrm{Ti}$ and ${ }^{56} \mathrm{Ni}$ in a Three-dimensional Supernova Model Resembling Cassiopeia A. Astrophys. J. 842, 13. doi:10.3847/1538-4357/aa72de.

Zanardo, G., Staveley-Smith, L., Ball, L., Gaensler, B.M., Kesteven, M.J., Manchester, R.N., Ng, C.Y., Tzioumis, A.K., Potter, T.M., 2010. Multifrequency Radio Measurements of Supernova 1987A Over 22 Years. Astrophys. J. 710, 1515-1529. doi:10.1088/0004-637X/710/2/1515. 